\title{
Management of laryngopharyngeal reflux with proton pump inhibitors
}

\author{
Christina Reimer \\ Peter Bytzer \\ Department of Medical \\ Gastroenterology, Køge University \\ Hospital, Denmark
}

\begin{abstract}
There is a lack of consistent guidelines and consensus for the diagnosis of laryngopharyngeal reflux (LPR). A therapeutic trial with a proton pump inhibitor (PPI) has been suggested to identify patients with LPR. This review focuses on the current difficulties in diagnosing the disease and examines the evidence for the effectiveness of PPI therapy in suspected reflux-related laryngeal symptoms. Additionally, mode of action, safety, and tolerability of PPIs are described. A total of 7 placebo-controlled trials were identified and included in the review. All studies evaluated the effect of a PPI on symptoms and objective laryngoscopic findings in suspected LPR. Data from these trials show that PPI therapy is no more effective than placebo in producing symptom relief in patients suspected of LPR. Symptoms, laryngoscopic findings, or abnormal findings on $\mathrm{pH}$ monitoring will not predict response to PPI therapy. High placebo response levels suggest a much more complex and multifactorial pathophysiology of LPR than simple acid reflux. Further studies are needed to characterize subgroups of patients with reflux-associated laryngeal symptoms that might benefit from treatment with PPI. Future studies should use validated patient reported outcome measures with endpoints that represent a predefined clinically meaningful change in symptom scores.
\end{abstract}

Keywords: laryngopharyngeal reflux, proton pump inhibitor

\section{Introduction}

A causal association between reflux of acidic gastric contents and symptoms and signs of laryngopharyngitis is plausible given the close anatomical relationship between the oesophagus and the hypopharynx and larynx. Refluxed material from the stomach, including acid and pepsin, may lead to direct chemical injuries and inflammation of the mucosa of the laryngopharyngeal structures, or may indirectly stimulate vagal afferents in the oesophagus. This is often referred to as reflux laryngitis or laryngopharyngeal reflux (LPR). It is estimated that 4\%-10\% of patients who consult ENT specialists do so because of complaints related to gastro-oesophageal reflux.

An association between gastro-oesophageal reflux symptoms and laryngeal symptoms such as hoarseness, cough, globus sensation, throat clearing, laryngitis and pharyngitis is supported by observations of frequent occurrence of these symptoms in patients with gastro-oesophageal reflux disease (GERD). In an Italian study, 74.4\% of GERD patients had at least one extra-oesophageal symptom and throat symptoms were reported by $19.9 \%-38.7 \%$ of the patients (Dore et al 2007).

There is a lack of consistent guidelines and consensus for the diagnosis of LPR (Ahmed et al 2006). The most common symptoms used to diagnose LPR include globus, throat clearing, cough, hoarseness, sore or burning throat, dysphagia, and dysphonia (Vaezi et al 2003). However, these symptoms are not specific for reflux induced damage and can also be associated with smoking, voice abuse, allergies, and viral infections. Prior reports have shown that less than $30 \%$ of patients with extraoesophageal manifestations of reflux have endoscopic evidence of oesophagitis (Vaezi et al 2003; Ahmed et al 2006). 
The most useful laryngeal signs for LPR are reported to be erythema, oedema, presence of a posterior commisure bar and cobble stoning (Ahmed, Khandwala, Abelson et al 2006). Recent data have documented a high intra- and interobserver variability of laryngeal examination, making the laryngoscopic diagnosis of LPR highly subjective. This increases the likelihood that many patients diagnosed with LPR based on objective findings may actually not have the disease (Branski et al 2002). Furthermore, generally accepted laryngoscopic signs of LPR can be found in up to $70 \%$ of healthy, normal volunteers (Hicks et al 2002). Thus, laryngoscopic findings are neither specific nor sensitive in the diagnosis of LPR.

Even though dual-channel pharyngo-oesophageal 24-h $\mathrm{pH}$ monitoring is considered the diagnostic gold standard for LPR by some (Noordzij et al 2001; Habermann et al 2002) the role of $\mathrm{pH}$ testing in the diagnosis of LPR remains controversial (Vaezi et al 2003). Proximal oesophageal and hypopharyngeal $\mathrm{pH}$ testing are not widely available and are considered less useful by both community and academic gastroenterologists (Ahmed et al 2006), and there is a lack of consensus on how much reflux in the hypopharynx is normal. Most studies show that hypopharyngeal $\mathrm{pH}$-monitoring is not a predictor of response to acid inhibitory therapy as response to therapy is no more likely in individuals with abnormal hypopharyngeal acid reflux compared to individuals with no acid reflux (El-Serag et al 2001; Noordzij et al 2001; Vaezi et al 2003; Williams et al 2004; Vaezi et al 2006; Wo et al 2006). Finally it should be taken into account that oesophageal pH-monitoring is not even a perfect gold standard test for GERD. Contrary to this view, Haberman et al (2002) found that patients with a positive $\mathrm{pH}$ monitoring had significant improvement in all symptoms, whereas in patients with a negative $\mathrm{pH}$ monitoring no statistically significant change was seen after open label pantoprazole. From this result, the authors argued that empirical acid inhibitory therapy serves to select patients with reflux-related problems from those without detectable reflux. This conclusion may be seriously flawed, though. The decrease in symptom scores was the same for both groups and the apparent difference in statistical outcomes was related to a small number of patients $(n=7)$ in the negative $\mathrm{pH}$ monitoring group.

A therapeutic trial with a proton pump inhibitor (PPI) has been suggested as a useful and cost-effective way of identifying patients with true reflux-related laryngopharyngeal symptoms (Ahmed et al 2006). A number of uncontrolled patient series have suggested that a positive response to empiric PPI therapy for 2-3 months will reliably establish a diagnosis of LPR. This review examines the evidence for the current recommendations of PPI therapy of suspected LPR.

\section{Patient reported outcomes in laryngopharyngeal reflux}

Apparently, 25\% of LPR patients experience spontaneous symptom resolution and $50 \%$ have a chronic course with intermittent exacerbations and remissions (Ford 2005), stressing the importance of double-blind, controlled evaluations using validated symptom response instruments.

In contrast to GERD, in which heartburn is the primary symptom, there is no predominant symptom for LPR. Accordingly, most studies have evaluated a number of symptoms or focused on changes in a single predominant throat symptom identified by the patient.

A number of composite symptom scores have been introduced but they have not been adequately validated. Various modifications of the Laryngeal Symptom Score (LSS) have been used in some studies (Williams et al 2004; Wo et al 2006; Qua et al 2007). The frequency and severity of a number (usually up to 6) of LPR symptoms are assessed over the preceding week to give a cumulative score.

The Reflux Symptom Index asks patients to grade 8 different throat symptoms and heartburn on a 6-graded scale (Belafsky et al 2002). This questionnaire has not been validated as a tool to identify patients with reflux induced laryngopharyngeal symptoms. A reflux symptom questionnaire has been modified to include laryngopharyngeal questions and was used in the study by Steward et al (2004).

Presence of moderate-to-severe GERD disqualified patients in some studies (Havas et al 1999; Vaezi et al 2006). Excluding patients with frequent heartburn or severe esophagitis might be problematic as the study population may not reflect daily clinical practice. On the other hand, there is concern over the ethical aspects of allocating patients with moderate to severe GERD to a placebo treatment arm. Furthermore, a high withdrawal rate might jeopardize the interpretation of the trial.

\section{Health-related quality of life}

The laryngopharyngeal reflux-health-related quality of life (LPR-HRQL) questionnaire is a 43-item instrument which assesses functional consequences of symptoms related to reflux laryngitis on the patients' daily life over the previous 4 weeks. The reliability, validity and responsiveness of the instrument has been tested (Carrau et al 2004; Carrau et al 2005). The questionnaire was found responsive to intervention 4 and 6 months into therapy. Unfortunately, earlier 
effects of therapy were not tested. The instrument has not been used in controlled PPI trials.

\section{Other outcome measures in laryngopharyngeal reflux Laryngoscopic findings}

All controlled PPI trials included patients with laryngoscopic findings compatible with LPR, findings that were evaluated with a new laryngoscopic examination at the end of therapy.

Most studies used a composite score based on gradings of laryngoscopic signs such as oedema, erythema, granuloma, ulcer, and amount of mucus as an outcome measure. Clinically relevant changes in these scores were not determined beforehand.

The precise correlation between laryngitis and symptoms remains unclear. Endoscopic signs of laryngitis may improve despite no discernible changes in symptom status (Williams et al 2004). On the other hand, a study has shown that the physical findings of LPR resolve more slowly than the symptoms (Belafsky 2001).

\section{$\mathrm{pH}$ monitoring}

Most PPI studies included a dual-channel $\mathrm{pH}$-monitoring at entry but only one study repeated the examination at the end of therapy (Wo et al 2006).

\section{Other outcome measures}

Other outcome measures have included changes in lifestyle factors related to reflux, changes in the SF-36 generic quality of life questionnaire and changes in voice quality as assessed by a computerized voice analysis (Steward et al 2004; Williams et al 2004).

\section{Mode of action and efficacy of proton pump inhibitors}

PPIs are substituted benzimidazoles. After oral administration they are absorbed in the small intestine as prodrugs and accumulate in the acidic environment of the canaliculi of the parietal cells where they are protonated and converted to an active and relatively stable sulfenamide form. This binds irreversibly to specific subunits on the outside surface of the luminal $\mathrm{H}^{+} / \mathrm{K}^{+}$-ATPase. As the final step in acid secretion involves activation of this enzyme, PPI therapy will reduce gastric acidity by inhibiting both basal and activated acid secretion (Horn 2000).

Five PPIs are currently widely available: esomeprazole, lansoprazole, omeprazole, pantoprazole, and rabeprazole.
The last four compounds are racemic isomer mixtures, whereas esomeprazole includes only the $\mathrm{S}$ isomer of omeprazole. There are subtle structural differences between the various PPIs that may affect aspects of their antisecretory activity and clinical utility (Bytzer 2006).

Once-daily dosing in the morning is more effective than dosing in the evening for all PPIs with respect to the suppression of intragastric acidity and daytime gastric acid secretion in particular. This may result from a better bioavailability being achieved with the morning dose. When higher doses are needed, these drugs should be given twice daily to achieve the optimal suppression of intragastric acidity. On twice-a-day dosing, inhibition of acid secretory capacity improves to $80 \%$ of maximally stimulated output (Sachs et al 2000).

PPIs are the drugs of choice for the treatment of GERD. They are highly effective in healing erosive GERD and in long-term resolution of acid-related symptoms (Jones and Bytzer 2001). Clinical data suggest that the optimal daily dose of PPIs for acute treatment of reflux-related symptoms and mucosal damage is about $30-40 \mathrm{mg}$. In less severe cases and in maintenance therapy doses of 10-20 mg daily may be sufficient (Kromer et al 1999). Standard-dose PPIs heal erosive esophagitis in more than $90 \%$ of patients after 8 weeks of treatment and achieve high symptom response rates compared with $\mathrm{H}_{2}$-receptor antagonists (Chiba et al 1997).

All PPIs are very safe drugs with an adverse event profile not different to placebo (Reilly 1999). The extensive clinical experience with PPIs has shown these agents to be well tolerated with a low risk of clinically relevant drug-drug interactions (Gerson and Triadafilopoulos 2001).

PPIs are considered the mainstay of therapy in LPR although there is controversy regarding their efficacy.

\section{Results from open-label trials}

Recommendations of treatment with PPIs in suspected LPR are based on the results of a number of open label studies (Kamel et al 1994; Hanson 1995; Jaspersen et al 1996; Habermann et al 1999; Shaw and Searl 1997; Wo et al 1997; Ulualp et al 2001; Delgaudio and Waring 2003; Park et al 2005; Siupsinskiene et al 2007). These are all uncontrolled studies investigating the effect of a PPI at varying doses on symptoms and laryngeal findings with treatment lasting from 6 to 24 weeks. In all studies there was a statistically significant improvement of both laryngeal symptoms and laryngoscopic findings at the end of therapy. The clinically significant measure for symptom improvement was defined in only 5 (Wo; Delgaudio; Park; Hanson; Jaspersen) of the 8 trials summarized in Table 1. The remaining 3 trials (Kamel; Habermann; Shaw) reported 
changes in composite symptom scores after therapy as proof of therapeutic effect. The clinical relevance of such overall change might be questioned.

Symptoms were rated by a number of different nonvalidated questionnaires with remarkably high and variable response rates ranging from $60 \%$ to $100 \%$.

\section{Results from placebo-controlled trials}

Six double-blinded placebo-controlled parallel studies and one placebo-controlled crossover trial were identified. All studies evaluated the effect of a PPI on symptoms and objective laryngoscopic findings. Ambulatory $\mathrm{pH}$ monitoring was performed in all studies, however abnormal results were used as inclusion criteria in only three studies (Noordzij et al 2001; Eherer et al 2003; Wo et al 2006). In only one study oesophageal symptoms of gastroesophageal reflux disease (heartburn) were specifically defined as an exclusion criteria (Vaezi et al 2006). In all other studies these symptoms were not required to enter nor did they exclude patients from participation.
The first published double-blinded placebo-controlled trial was a study by Havas et al (1999). Twenty patients with symptoms and laryngoscopic signs of LPR presenting to one single otolaryngologist were randomized to treatment with lansoprazole $30 \mathrm{mg}$ bid or placebo for 12 weeks. Patients with pre-existing anti-secretory therapy or severe reflux oesophagitis at endoscopy were excluded. Patients were investigated with upper GI endoscopy, oesophageal manometry, and dual $\mathrm{pH}$ probe monitoring. The presence of typical reflux symptoms were registered at study entry. Severity and frequency of throat symptoms and laryngoscopic findings were evaluated at baseline, and after 6 and 12 weeks. Data from 15 patients were available for analysis.

Symptoms and laryngoscopic findings improved in both groups with no significant differences between lansoprazole and placebo. Even when data were analyzed based on the results of the $\mathrm{pH}$ monitoring no differences between placebo and active drug could be discerned.

In a study by Wo et al (2006) 39 patients with symptoms of LPR and a positive triple-sensor $\mathrm{pH}$ test were randomized after a 2 -week run-in to double-blind treatment

Table I Summary of open-label trials assessing effects of PPI therapy on symptoms of LPR

\begin{tabular}{|c|c|c|c|c|c|}
\hline Study & $\begin{array}{l}\text { No of } \\
\text { patients }\end{array}$ & Inclusion criteria & Medication & $\begin{array}{l}\text { Duration of } \\
\text { treatment } \\
\text { (wk) }\end{array}$ & Outcome measure \\
\hline Kamel (1994) & 16 & $\begin{array}{l}\text { Persisting LPR symptoms } \\
\text { and signs despite prior } \\
\text { H2RA treatment }\end{array}$ & $\begin{array}{l}\text { Omeprazole } 40 \mathrm{mg} \text { od, } \\
\text { increased to } 40 \mathrm{mg} \text { bid } \\
\text { in } 6 \text { pt.s with ongoing } \\
\text { symptoms }\end{array}$ & $6-24$ & $\begin{array}{l}\text { Unspecified change from } \\
\text { baseline in mean composite } \\
\text { symptom score }\end{array}$ \\
\hline Hanson (1995) & 41 & $\begin{array}{l}\text { LPR symptoms and signs } \\
\text { unresponsive to nocturnal } \\
\text { anti-reflux precautions }\end{array}$ & Omeprazole $40 \mathrm{mg}$ od & 4 & Resolution of symptoms \\
\hline Jaspersen (1996) & 21 & $\begin{array}{l}\text { LPR symptoms and signs } \\
\text { and oesophagitis at upper } \\
\mathrm{Gl} \text { endoscopy }\end{array}$ & Omeprazole $40 \mathrm{mg}$ od & 4 & Resolution of symptoms \\
\hline Shaw (1997) & 96 & LPR symptoms & Omeprazole $20 \mathrm{mg}$ od & 12 & $\begin{array}{l}\text { Unspecified improvement in } \\
\text { symptoms }\end{array}$ \\
\hline Wo (1997) & 21 & LPR symptoms and signs & Omeprazole $40 \mathrm{mg}$ od & 8 & $\begin{array}{l}\text { Resolution of symptoms or } \\
\text { patient reported satisfactory } \\
\text { effect of treatment }\end{array}$ \\
\hline Habermann (1999) & 29 & $\begin{array}{l}\text { Voice disorders and } \\
\text { symptoms of LPR }\end{array}$ & Pantoprazole $40 \mathrm{mg}$ od & 6 & $\begin{array}{l}\text { Unspecified change from } \\
\text { baseline in mean composite } \\
\text { symptom score }\end{array}$ \\
\hline Delgaudio (2003) & 30 & LPR symptoms & Esomeprazole $40 \mathrm{mg}$ od & 8 & $\begin{array}{l}\text { Reduction of at least } 50 \% \text { in } \\
\text { symptom score and remaining } \\
\text { symptoms no morte than mild } \\
\text { in severity }\end{array}$ \\
\hline Park (2005) & 85 & LPR symptoms and signs & $\begin{array}{l}\text { Lansoprazole } 30 \mathrm{mg} \text { bid }(n=30) \text {, } \\
\text { omprazole } 20 \mathrm{mg} \text { bid }(n=30) \\
\text { esomprazole } 40 \mathrm{mg} \text { od }(n=25)\end{array}$ & 16 & $\begin{array}{l}\text { Reduction of at least } 50 \% \text { in } \\
\text { primary symptom score }\end{array}$ \\
\hline
\end{tabular}

Abbreviations: LPR, laryngopharyngeal reflux; PPI, proton pump inhibitor. 


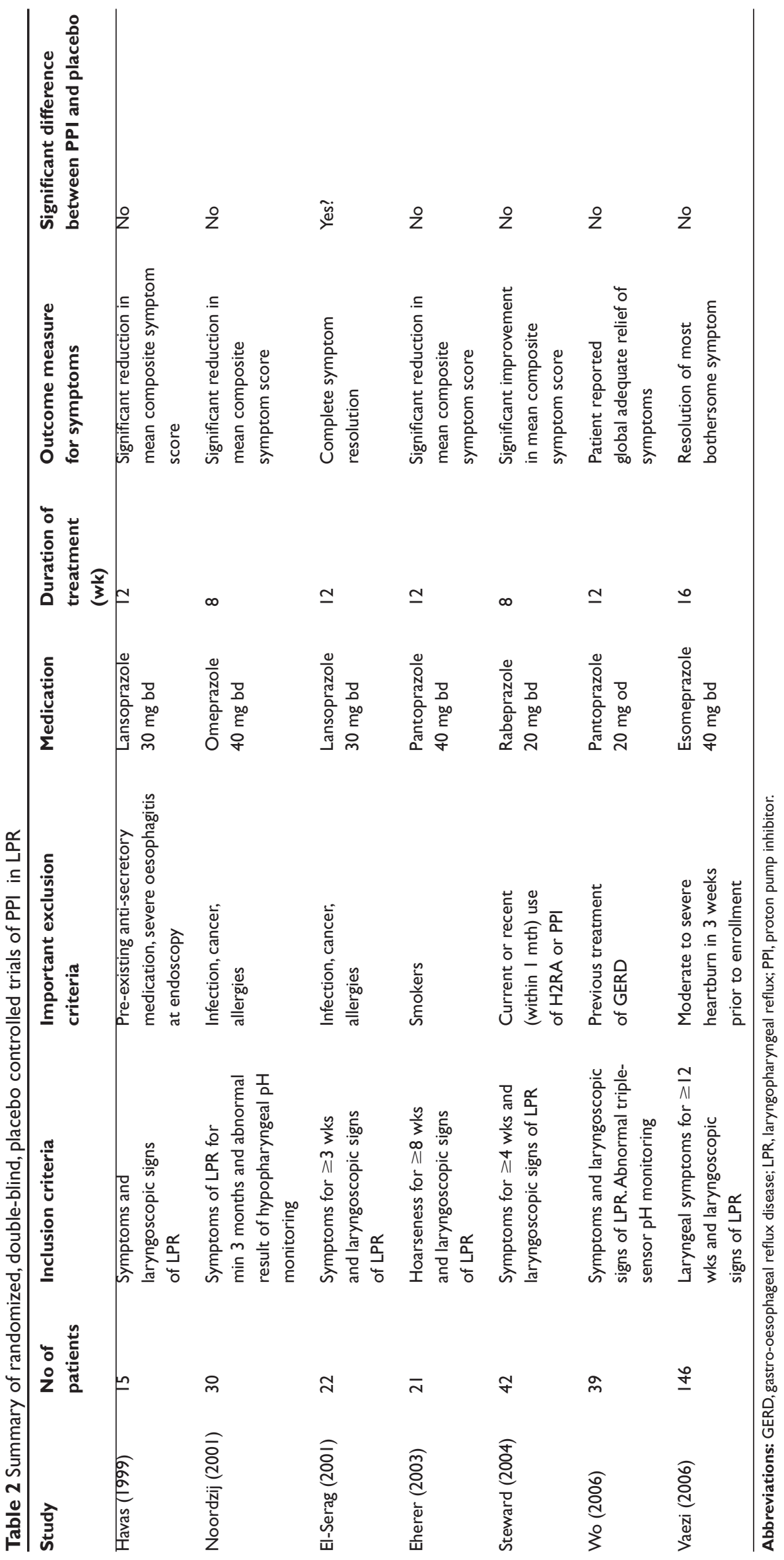


with pantoprazole 20 once daily or placebo for 12 weeks followed by a 4-week follow-up of treatment. Laryngeal findings were scored using the Reflux Finding Score prior to enrolment and after treatment. The triple-sensor $\mathrm{pH}$ test was repeated in week 12 on treatment to confirm acid suppression. Patients scored the severity of both laryngeal and oesophageal symptoms weekly and assessed if they had adequate relief of symptoms.

Symptoms improved during the treatment period in both study groups with no significant difference. Adequate relief by the end of the treatment period was reported by $40 \%$ in the pantoprazole group and $42 \%$ in the placebo group. There was no significant improvement in the Reflux Finding Score before and after treatment in subjects randomized to pantoprazole as well as no differences between pre- and post treatment hypopharyngeal reflux episodes in either group. There was no correlation between laryngeal findings or suppression of acid reflux and symptom improvement.

In a double-blinded placebo-controlled study by Noordzij et al (2001), patients with symptoms of LPR were investigated with a 24-hour dual-channel $\mathrm{pH}$ probe study. A total of 30 patients with a positive $\mathrm{pH}$ study were randomly assigned to treatment with omeprazole $40 \mathrm{mg}$ or placebo twice a day for 2 months. Laryngeal symptoms and laryngoscopic signs of LPR were recorded at study entry and after 1 and 2 months of treatment. Symptom scores were assessed by multiplying the number of days with symptoms over the past 2 weeks with the severity of each symptom rated on a visual analog scale from none to most severe.

Symptom scores improved over time for both treatment groups with no significant differences. By performing secondary analysis the authors were able to demonstrate a statistically significant improvement for 2 laryngeal symptoms (mild hoarseness and throat clearing) in the omeprazole group compared with the placebo group, but not for other LPR symptoms, nor for heartburn. There was no significant change in endoscopic laryngeal findings in either group.

In a placebo-controlled crossover trial (Eherer et al 2003) 21 patients with symptoms suggestive of laryngitis and abnormal oesophageal or pharyngeal $\mathrm{pH}$-study were randomized to treatment with pantoprazole $40 \mathrm{mg}$ bid or placebo for 3 months, each separated by a 2 -week washout period. Patients scored intensity and frequency of both laryngeal and oesophageal symptoms and were scored for laryngeal changes at baseline and at the end of each 3 months treatment period. By the end of the 2-week wash-out period, 14 of the 21 patients underwent a second $\mathrm{pH}$-study.
There were no significant changes in symptom scores after the first treatment period within or between the groups for either oesophageal or laryngeal symptoms. After reversal of treatments there was no significant change in symptom scores in any group. A significant decrease in laryngeal scores after 3 months of treatment was seen in both the pantoprazole and the placebo group. This decrease was maintained in both groups after switch of treatment.

In another double-blind, randomized placebo-controlled trial (Steward et al 2004) 42 patients with laryngeal symptoms and objective findings suggestive of LPR underwent a distal and proximal oesophageal 24-h pH monitoring. Questionnaires with assessment of frequency and severity of typical reflux symptoms and laryngeal symptoms as well as SF-36 were filled out prior to randomization to 2 months treatment with rabeprazole $20 \mathrm{mg}$ bid or placebo and by the end of the treatment period. In addition, patients in both groups were instructed on relevant lifestyle modifications. Outcome measures included change in total symptom scores and proportion of subjects with significant global improvement.

Both rabeprazole- and placebo-treated patients demonstrated significant improvement in total symptom scores with no statistically significant differences between the two groups.

The largest study to date is a multicenter, double-blind, placebo-controlled study by Vaezi et al (2006). A total of 146 patients with laryngeal symptoms and laryngoscopic signs consistent with laryngopharyngeal reflux were randomized in a 2:1 ratio to esomeprazole $40 \mathrm{mg}$ or placebo twice a day for 16 weeks. Patients with moderate to severe heartburn in the preceding 3 weeks were excluded. Before randomization patients underwent a 7- to 14-day screening period with daily registration of 5 different laryngeal symptoms. By the end of the screening period patients identified their single most bothersome symptom. To be eligible for randomization patients were required to report at least moderately severe symptoms for a minimum of 3 days. Before receiving study medication patients were offered a pharyngo-oesophageal $\mathrm{pH}$ monitoring with 3 probes proximally and distally in the oesophagus and in the hypopharynx. Additionally patient quality of life was assessed using the laryngopharyngeal reflux-health-related quality of life (LPR-HRQL) questionnaire at baseline and at the final visit. Patients reported daily symptoms on a diary card throughout the treatment period. The primary outcome measure was the percentage of patients who had resolution of the most bothersome symptom at the final visit. Resolution of symptoms was defined as a severity score of 0 (none) during 
the last 7 days of the study, allowing symptoms of minimal severity for up to 3 days.

Resolution of the primary symptom was obtained in $14.7 \%$ and $16.0 \%$ in the esomeprazole and placebo groups, respectively with no statistically significant difference. For secondary outcome measures, such as improvement in laryngeal signs, relief of primary symptom and improvement in LPR-HRQOL, there was no difference between the treatment groups.

The only double-blind, randomized placebo-controlled study that claims a statistically significant effect of a PPI on laryngeal symptoms was performed by El-Serag and coworkers (El-Serag et al 2001). Of 27 patients diagnosed with idiopathic chronic laryngitis, who were referred to endoscopy and a 24-hour $\mathrm{pH}$ study from one single otolaryngologist, a total of 22 patients were randomized to 3 months treatment with lansoprazole $30 \mathrm{mg}$ bid or placebo. Oesophageal and laryngeal symptoms were scored at entry, after 6 weeks of treatment and at the end of the study. Additionally, laryngeal findings were evaluated prior to enrolment and after treatment. High-grade laryngoscopic findings suggestive of reflux, such as contact ulcers and granuloma, were found in 5 and 1 of the 12 patients in the lansoprazole group and in 1 and 0 of the 10 patients in the placebo group, respectively. Two patients dropped out after randomization leaving 11 and 9 patients in the lansoprazole and placebo groups, respectively.

The primary outcome measure was complete symptom resolution for laryngeal symptoms, which was found in 6/12 patients $(50 \%)$ in the lansoprazole group. According to the authors, this was significantly higher than the response rate of $10 \%(1 / 10)$ in the placebo group. However, a two-sided Fisher's exact test reveals a non-significant p-value of 0.069 for this difference. Restricting the analysis to the 20 per protocol patients does not improve the p-value, which remains insignificant at $\mathrm{p}=0.074$ when applying a correct non-parametric test, which adjusts for small cell size. Baseline oesophageal and laryngeal examinations, signs and symptoms did not predict response to therapy.

\section{Results from meta-analyses}

Qadeer et al (2006) performed a meta-analysis of eight randomized controlled trials, including a small study from 2001 which remains published only in abstract form (Langevin and Hanh 2001). Study results were pooled and analyzed in order to estimate the overall efficacy of PPI therapy in suspected LPR. The proportion of patients in each study who reported $\geq 50 \%$ reduction in laryngeal symptoms compared with baseline was abstracted. Data from the studies were also analyzed to identify potential factors that could be associated with this primary outcome.

Pooled absolute rates of response, defined as $\geq 50 \%$ reduction in laryngeal symptoms, in the PPI and placebo groups were 50\% (98/195) and 41\% (61/149), respectively, which represent a non-significant symptom reduction with PPI compared with placebo. No significant clinical predictors of response to therapy were identified. A funnel plot analysis showed evidence of publication bias with two studies outside the funnel; both these studies (El-Serag et al 2001; Langevin and Hanh 2001) claimed a significant PPI benefit.

Another recent meta-analysis by Gatta et al (2007) analyzed 5 studies with a total of 247 patients, including a trial on chronic cough patients with no data on laryngeal examinations (Ours et al 1999). Four of these studies were also included in the meta-analysis by Qadeer. The pooled relative risk (RR) of reporting symptomatic improvement or resolution of symptoms and the proportion of patients responding to active treatment or placebo was calculated as well as the number needed to treat (NNT).

With a pooled RR of 1.18 , a non-significant difference in response rate of $3.6 \%$ between the treatment group (25\%) and the placebo group (21.4\%) and a NNT of 53, the authors concluded that treatment with PPIs is no more effective than placebo in resolving or improving laryngo-pharyngeal symptoms presumed to be due to GERD. Funnel plot analysis showed an asymmetry suggesting the potential for publication bias or small study effects.

\section{Conclusions}

Data from controlled treatment trials convincingly show that PPI therapy is no more effective than placebo in producing symptom relief in patients suspected of laryngo-pharyngeal reflux disease. Furthermore, neither symptoms, nor laryngoscopic findings or abnormal findings on $\mathrm{pH}$ monitoring will predict response to PPI therapy. A reliable diagnostic test for LPR or one that might predict response to a PPI does not exist.

Surprisingly, empiric PPI therapy for 2-3 months is still recommended in clinical reviews and guidelines as the most cost-effective and useful approach to the initial diagnosis of LPR (Delgaudio and Waring 2003; Ford 2005; Dore et al 2007). Because of the high placebo response rates seen in the clinical trials a positive outcome of therapy in daily clinical practice should only cautiously be taken as proof of an established association between acid reflux and laryngeal symptoms. Therefore, PPI therapy should always be followed by withdrawal in order to determine whether a possible effect 
is actually due to acid suppression or represents a placebo response or spontaneous resolution of symptoms.

The relative inefficacy of PPI treatment also challenges the current diagnosis pattern of LPR based on unspecific symptoms and findings and a controversial role of $\mathrm{pH}-$ monitoring. This may lead to overdiagnosis of the disease with disappointing outcome of acid suppressive therapy as a result. Especially laryngoscopic findings seem to be difficult to interpret with high inter- and intra-observer variability leading to uncertain correlation between symptoms, endoscopic signs and response to therapy.

The results from the high quality study by Vaezi et al (Vaezi, Richter, Stasney et al 2006) with very low response rates and without significant difference in response between PPI therapy and placebo makes it reasonable to conclude that patients without typical symptoms of GERD (heartburn) will not benefit from PPI therapy. Unlike with GERD, response to PPI therapy in patients with LPR has been highly variable. Some have argued that this is in part because LPR patients require higher doses and prolonged therapy compared to GERD patients. Several experts have recommended that the empiric therapy has to be aggressive with a period of treatment of at least 2-3 months. However, the results from placebo-controlled double-blind trials and from the metaanalyses suggest that lack of response to empiric PPI should not lead to an escalation of dose or treatment length, but rather to a revised diagnosis.

The placebo response rates in most clinical trials were remarkably higher than the placebo response rates generally seen in randomized trials estimating the symptomatic effects of PPI therapy in patients with GERD, and were at a level comparable to that seen in functional GI disorders. This indicates that the causes of LPR are much more complex and multifactorial than can be explained by the simple chemical injury associated with refluxed stomach contents. This calls for further studies to help characterize subgroups of patients with reflux-associated laryngeal symptoms that might benefit from treatment with a PPI. Studies that utilize validated patient reported outcome measures with endpoints that represent a predefined clinically meaningful change in symptom scores are needed.

\section{References}

Ahmed TF, Khandwala F, Abelson TI, et al. 2006. Chronic laryngitis associated with gastroesophageal reflux: prospective assessment of differences in practice patterns between gastroenterologists and ENT physicians. Am J Gastroenterol, 101:470-8.

Belafsky PC, Postma GN, Koufman JA. 2001. Laryngopharyngeal reflux symptoms improve before changes in physical findings. Laryngoscope, 111:979-81.
Belafsky PC, Postma GN, Koufman JA. 2002. Validity and reliability of the reflux symptom index (RSI). $J$ Voice, 16:274-7.

Branski RC, Bhattacharyya N, Shapiro J. 2002. The reliability of the assessment of endoscopic laryngeal findings associated with laryngopharyngeal reflux disease. Laryngoscope, 112:1019-24.

Bytzer P. 2006. Introduction: pharmacodynamic and pharmacokinetic properties of proton pump inhibitors and their clinical impact - focus on rabeprazole. Aliment Pharmacol Ther symp ser, 2:311-3.

Carrau RL, Khidr A, Crawley JA, et al. 2004. The impact of laryngopharyngeal reflux on patient-reported quality of life. Laryngoscope, 114:670-4.

Carrau RL, Khidr A, Gold KF, et al. 2005. Validation of a quality-of-life instrument for laryngopharyngeal reflux. Arch Otolaryngol Head Neck Surg, 131:315-20.

Chiba N, De Gara CJ, Wilkinson JM, et al. 1997. Speed of healing and symptom relief in grade II to IV gastroesophageal reflux disease: a meta-analysis. Gastroenterology, 112:1798-810.

Delgaudio JM, Waring JP. 2003. Empiric esomeprazole in the treatment of laryngopharyngeal reflux. Laryngoscope, 113:598-601.

Dore MP, Pedroni A, Pes GM, et al. 2007. Effect of antisecretory therapy on atypical symptoms in gastroesophageal reflux disease. Dig Dis Sci, 52:463-8.

Eherer AJ, Habermann W, Hammer HF, et al. 2003. Effect of pantoprazole on the course of reflux-associated laryngitis: a placebo-controlled double-blind crossover study. Scand J Gastroenterol, 38:462-7.

El-Serag HB, Lee P, Buchner A, et al. 2001. Lansoprazole treatment of patients with chronic idiopathic laryngitis: a placebo-controlled trial. Am J Gastroenterol, 96:979-83.

Ford CN. 2005. Evaluation and management of laryngopharyngeal reflux. JAMA, 294:1534-40

Gatta L, Vaira D, Sorrenti G, et al. 2007. Meta-analysis: the efficacy of proton pump inhibitors for laryngeal symptoms attributed to gastrooesophageal reflux disease. Aliment Pharmacol Ther, 25:385-92.

Gerson LB, Triadafilopoulos G. 2001. Proton pump inhibitors and their drug interactions: an evidence-based approach. Eur J Gastroenterol Hepatol, 13:611-6.

Habermann W, Eherer A, Lindbichler F, et al. 1999. Ex juvantibus approach for chronic posterior laryngitis: results of short-term pantoprazole therapy. J Laryngol Otol, 113:734-9.

Habermann W, Kiesler K, Eherer A, et al. 2002. Short-term therapeutic trial of proton pump inhibitors in suspected extraesophageal reflux. $J$ Voice, 16:425-32.

Hanson DG, Kamel PL, Kahrilas PJ. 1995. Outcomes of antireflux therapy for the treatment of chronic laryngitis. Ann Otol Rhinol Laryngol, 104:550-5.

Havas T, Huang S, Levy M. 1999. Double blind randomized placebocontrolled trial of proton pump inhibitor therapy. Aust J Otolaryngol, 3:243-6.

Hicks DM, Ours TM, Abelson TI, et al. 2002. The prevalence of hypopharynx findings associated with gastroesophageal reflux in normal volunteers. $J$ Voice, 16:564-79.

Horn J. 2000. The proton-pump inhibitors: similarities and differences. Clin Ther, 22:266-80.

Jaspersen D, Weber R, Hammar CH, et al. 1996. Effect of omeprazole on the course of associated esophagitis and laryngitis. J Gastroenterol, $31: 765-7$.

Jones R, Bytzer P. 2001. Review article: acid suppression in the management of gastro-oesophageal reflux disease--an appraisal of treatment options in primary care. Aliment Pharmacol Ther, 15:765-72.

Kamel PL, Hanson D, Kahrilas PJ. 1994. Omeprazole for the treatment of posterior laryngitis. Am J Med, 96:321-6.

Kromer W, Horbach S, Luhmann R. 1999. Relative efficacies of gastric proton pump inhibitors: their clinical and pharmacological basis. Pharmacology, 59:57-77.

Langevin S, Hanh N. 2001. GERD-induced ENT symptoms: A prospective placebo controlled study with omeprazole $40 \mathrm{mg}$ a day. Gastroenterology, 120:A16. 
Noordzij JP, Khidr A, Evans BA, et al. 2001. Evaluation of omeprazole in the treatment of reflux laryngitis: a prospective, placebo-controlled, randomized, double-blind study. Laryngoscope, 111:2147-51.

Ours TM, Kavuru MS, Schilz RJ, et al. 1999. A prospective evaluation of esophageal testing and a double-blind, randomized study of omeprazole in a diagnostic and therapeutic algorithm for chronic cough. Am J Gastroenterol, 94:3131-8.

Park W, Hicks DM, Khandwala F, et al. 2005. Laryngopharyngeal reflux: prospective cohort study evaluating optimal dose of proton-pump inhibitor therapy and pretherapy predictors of response. Laryngoscope, $115: 1230-8$.

Qadeer MA, Phillips CO, Lopez AR, et al. 2006. Proton pump inhibitor therapy for suspected GERD-related chronic laryngitis: a meta-analysis of randomized controlled trials. Am J Gastroenterol, 101:2646-54.

Qua CS, Wong CH, Gopala K, et al. 2007. Gastro-oesophageal reflux disease in chronic laryngitis: prevalence and response to acid-suppressive therapy. Aliment Pharmacol Ther, 25:287-95.

Reilly JP. 1999. Safety profile of the proton-pump inhibitors. Am J Health Syst Pharm, 56:S11-S17.

Sachs G, Shin JM, Munson K, et al. 2000. Review article: the control of gastric acid and Helicobacter pylori eradication. Aliment Pharmacol Ther, 14:1383-401.

Shaw GY, Searl JP. 1997. Laryngeal manifestations of gastroesophageal reflux before and after treatment with omeprazole. South Med J, 90:1115-22.
Siupsinskiene N, Adamonis K, Toohill RJ. 2007. Quality of life in laryngopharyngeal reflux patients. Laryngoscope, 117:480-4.

Steward DL, Wilson KM, Kelly DH, et al. 2004. Proton pump inhibitor therapy for chronic laryngo-pharyngitis: a randomized placebo-control trial. Otolaryngol Head Neck Surg, 131:342-50.

Ulualp SO, Toohill RJ, Shaker R. 2001. Outcomes of acid suppressive therapy in patients with posterior laryngitis. Otolaryngol Head Neck Surg, 124:16-22.

Vaezi MF, Hicks DM, Abelson TI, et al. 2003. Laryngeal signs and symptoms and gastroesophageal reflux disease (GERD): a critical assessment of cause and effect association. Clin Gastroenterol Hepatol, $1: 333-44$.

Vaezi MF, Richter JE, Stasney CR, et al. 2006. Treatment of chronic posterior laryngitis with esomeprazole. Laryngoscope, 116:254-60.

Williams RB, Szczesniak MM, Maclean JC, et al. 2004. Predictors of outcome in an open label, therapeutic trial of high-dose omeprazole in laryngitis. Am J Gastroenterol, 99:777-85.

Wo JM, Grist WJ, Gussack G, et al. 1997. Empiric trial of high-dose omeprazole in patients with posterior laryngitis: a prospective study. Am J Gastroenterol, 92:2160-5.

Wo JM, Koopman J, Harrell SP, et al. 2006. Double-blind, placebocontrolled trial with single-dose pantoprazole for laryngopharyngeal reflux. Am J Gastroenterol, 101:1972-8. 
